\title{
THE PROJECTIVE COVER OF THE TRIVIAL REPRESENTATION FOR A FINITE GROUP OF LIE TYPE IN DEFINING CHARACTERISTIC
}

\author{
SHIGEO KOSHITANI AND JÜRGEN MÜLLER
}

\begin{abstract}
We give a lower bound of the Loewy length of the projective cover of the trivial module for the group algebra $k G$ of a finite group $G$ of Lie type defined over a finite field of odd characteristic $p$, where $k$ is an arbitrary field of characteristic $p$. The proof uses Auslander-Reiten theory.
\end{abstract}

\section{INTRODUCTION}

One of the main problems in the modular representation theory of finite groups is obtaining classes of finite groups $G$ such that the group algebras $k G$ have specific ring-theoretical properties, where $k$ is a field of positive characteristic $p$, just as Brauer stated in [4, Problem 16]. Examples of such properties are the Loewy length $\operatorname{LL}\left(P\left(k_{G}\right)\right)$ of the projective cover $P\left(k_{G}\right)$ of the trivial $k G$-module $k_{G}$, and the 'first' Cartan invariant $c_{11}(G):=\left[P\left(k_{G}\right): k_{G}\right]$ of the group algebra $k G$, that is the multiplicity of $k_{G}$ as a composition factor of $P\left(k_{G}\right)$.

In the present paper, we are interested in the questions for representations of finite groups of Lie type in their defining characteristic; see also for example [12, (2.6)] and [13, Section 11.4]. In even characteristic we are in a good shape indeed: Let $G$ be a simple finite group of Lie type defined over a finite field of characteristic $p=2$. Then it follows from results of Okuyama 34 and Erdmann 8 that $\operatorname{LL}\left(P\left(k_{G}\right)\right)=3$ if and only if $G=\mathrm{SL}_{3}(2)$, see [22, Theorem 3.3]; and by [22, Theorem 1.2] we always have $\operatorname{LL}\left(P\left(k_{G}\right)\right) \neq 4$. Moreover, by [23, Lemma 4.5] we have $c_{11}(G)=2$ if and only if $G=\mathrm{SL}_{3}(2)$. Hence in conclusion for this class of groups we have $\operatorname{LL}\left(P\left(k_{G}\right)\right) \geq 5$ if and only if $c_{11}(G) \geq 3$ if and only if $G \neq \mathrm{SL}_{3}(2)$.

Here we are now interested in the odd characteristic case, and the purpose of this paper is to present the following theorem.

Theorem 1.1. Assume that $p$ is an odd prime, and $G$ is a simple or an almost simple finite group of Lie type (in the sense of 2.1) defined over a finite field of characteristic $p$, such that the Sylow p-subgroups of $G$ are non-cyclic, that is $G \notin$ $\left\{\mathrm{SL}_{2}(p),{ }^{2} G_{2}(\sqrt{3})^{\prime}\right\}$. Then the projective cover $P\left(k_{G}\right)$ of the trivial $k G$-module has Loewy length $\operatorname{LL}\left(P\left(k_{G}\right)\right) \geq 5$.

This sheds new light on a couple of our earlier results in [24]. Firstly, we get the following, providing an alternative proof of [24, Proposition 4.12]:

Date: March 22, 2022.

2000 Mathematics Subject Classification. 20C05,20C20,20C33.

Key words and phrases. Modular representations, projective cover of the trivial module, finite groups of Lie type in defining characteristic. 
Corollary 1.2. Under the same assumptions as in Theorem 1.1, the principal block algebra $B_{0}(G)$ of $k G$ has the Loewy length $\operatorname{LL}\left(B_{0}(G)\right) \geq 5$.

Secondly, our method of proof of Theorem 1.1 also allows to generalize 24, Proposition 4.10] (which was proved under the additional assumption $p \geq 5$ ):

Theorem 1.3. Let $p$ be an odd prime, let $G$ be a finite group such that $O_{p^{\prime}}(G)=$ $\{1\}$, and assume that the principal block algebra $B_{0}(G)$ of $k G$ has Loewy length $\operatorname{LL}\left(B_{0}(G)\right)=4$. Then $F^{*}(G)=E(G)=O^{p^{\prime}}(G)$ is a non-abelian simple group.

Our line of reasoning in proving the above results can be summarized as follows: Letting $G$ be a finite group, assume that $\operatorname{LL}\left(B_{0}(G)\right) \leq 4$. Then we have $\mathrm{LL}\left(P\left(k_{G}\right)\right) \leq 4$ as well, from which we conclude that the heart

$$
\mathcal{H}\left(k_{G}\right):=\operatorname{rad}\left(P\left(k_{G}\right)\right) / \operatorname{soc}\left(P\left(k_{G}\right)\right)
$$

of $P\left(k_{G}\right)$ actually is a simple $k G$-module $S$, where apart from a trivial exceptional case we have $S \neq k_{G}$. If now $G$ is a finite group of Lie type, we are in a position to apply the Kawata-Michler-Uno Theorem [20, which in turn is a specific application of Auslander-Reiten theory to this class of groups. Actually, to cover all the cases allowed by our assumptions we have to extend the Kawata-Michler-Uno Theorem slightly, which is done by unraveling the strategy of proof in [20, and checking the necessary conditions for the additional cases.

This paper thus is organized as follows: In Section 2 we collect the necessary prerequisites from the theory of finite groups of Lie type and their representations in defining characteristic. Moreover, we revisit the Kawata-Michler-Uno heorem and its proof, in order to extend it to the class of groups considered here. In Section 3 we then first prove the general statement on $\mathcal{H}\left(k_{G}\right)$ mentioned in the previous paragraph, and subsequently give proofs of our main results.

We would like to stress the fact that our approach stays entirely in the realm of modular representation theory, no results of ordinary representation theory or character theory are needed, not even behind the scenes to prove the KawataMichler-Uno Theorem. Moreover, apart from the case-by-case analysis needed to prove the latter, our approach is completely structural, without case distinctions.

1.4. Cartan invariants. In view of the comments on the even characteristic case at the beginning of this section, we have a quick look onto Cartan invariants:

(a) Let $p$ be odd and $G$ a finite group. Then, apart from the above-mentioned exception, we conclude that in general the condition $\operatorname{LL}\left(P\left(k_{G}\right)\right) \leq 4$ implies that the Cartan invariant $c_{11}(G)=2$. Hence the question arises whether the condition $\operatorname{LL}\left(P\left(k_{G}\right)\right) \geq 5$ implies $c_{11}(G) \geq 3$. But this is not true in general, in other words $c_{11}(G)=2$ is a strictly weaker condition than $\operatorname{LL}\left(P\left(k_{G}\right)\right) \leq 4$ :

One of the smallest counter-examples is $G:=C_{3}^{2}: Q_{8}$ for $p=3$, where $C_{3}$ and $Q_{8}$ are the cyclic group of order 3 and the quaternion group of order 8, respectively, and the action of $Q_{8}$ on the elementary abelian group $C_{3}^{2}$ is regular. Then we have $c_{11}(G)=2$, although $\operatorname{LL}\left(P\left(k_{G}\right)\right)=5$; actually, all projective indecomposable $k G$ modules in $B_{0}(G)$ have Loewy length 5 , so that $\mathrm{LL}\left(B_{0}(G)\right)=5$ as well; see also [25, Lemma (4.2) and Lemma (4.3)], where infinitely many such examples are given.

(b) The present paper arose out of an attempt to approach the question raised in our earlier paper [24, whether or not for any non-abelian finite simple group $G$ having non-cyclic Sylow $p$-subgroups, where $p$ is odd, the Cartan invariant $c_{11}(G) \geq$ 
3. Unfortunately, by the above comments, the condition $c_{11}(G)=2$ is strictly weaker than $\operatorname{LL}\left(P\left(k_{G}\right)\right) \leq 4$, so that after all Theorem 1.1 does not help here.

Moreover, this is also related to the recent result [29, Theorem 7.1] saying that, if $G$ is a classical simple finite group of Lie type, and $l$ is an odd prime such that the Sylow $l$-subgroups are non-cyclic, then the $l$-modular Cartan invariant $c_{11}^{(l)}(G) \geq 3$. But in the proof given there the defining characteristic case $l=p$ is erroneously attributed to [24, Proposition 4.12], which only states that the Loewy length of the block algebra $B_{0}(G)$ as a whole is $\operatorname{LL}\left(B_{0}(G)\right) \neq 4$, but nothing comprehensive is said about $P\left(k_{G}\right)$ or $c_{11}(G)$. Actually, when we were preparing the present manuscript, we noticed the above-mentioned gap, and informed the authors of [29 about it, and after this manuscript was completed we learned that they have been able to close the gap in [30. We would like to point out that [29, 30] make heavy use of ordinary character theory of finite groups of Lie type, so that their approach is fundamentally different from ours.

1.5. Notation and terminology. We shall in particular use the following notation. Let $k$ be an arbitrary field of positive characteristic $p$, and let $A$ be a finite-dimensional $k$-algebra. Unless stated otherwise we mean by an $A$-module a finitely generated right $A$-module. If $M$ is an $A$-module, we write $\operatorname{rad}(M)$ for the Jacobson radical of $M$, and $P(M)$ for the projective cover of $M$. We say that $n=\mathrm{LL}(M)$ is the Loewy length of $M$, if $n \geq 0$ is smallest with $\operatorname{rad}^{n}(M)=\{0\}$.

Moreover, if $G$ is a finite group, we write $Z(G)$ for the center of $G$, and $F(G)$ for the Fitting subgroup of $G$. We let $E(G)$ be the layer of $G$, that is the central product of the components of $G$, where a component of $G$ is a subnormal quasi-simple subgroup, and we write $F^{*}(G)=F(G) E(G)$ for the generalized Fitting subgroup of $G$. We denote by $k_{G}$ the trivial $k G$-module, and by $B_{0}(G):=B_{0}(k G)$ the principal block algebra of $k G$. Given a $k G$-module $M$, we write $M^{*}:=\operatorname{Hom}_{k}(M, k)$ for the dual module of $M$, which becomes a right $k G$-module with resepct to the contragredient acion, and $M$ is called self-dual if $M \cong M^{*}$ as $k G$-modules.

For other general notation and terminology we refer [33] and [36], as far as representation theory and finite group theory are concerned, respectively. Moreover, for the necessary background on finite groups of Lie type and Auslander-Reiten theory, we refer to [31] and [2, respectively.

\section{Groups of Lie type in Defining Characteristic}

We collect the facts needed from the theory of finite groups of Lie type and their representations.

2.1. Tits's Theorem. Let $\mathbf{G}$ be a simply-connected simple linear algebraic group over the algebraic closure $\overline{\mathbb{F}}_{p}$ of the field $\mathbb{F}_{p}$ with $p$ elements. Let $F: \mathbf{G} \rightarrow \mathbf{G}$ be a Steinberg endomorphism, see [31, Ch.21]. Let $\mathbf{T}$ be an $F$-stable maximal torus of $\mathbf{G}$, contained in an $F$-stable Borel subgroup $\mathbf{B}$ of $\mathbf{G}$, and let $\mathbf{U}$ be the unipotent radical of $\mathbf{B}$. Let $q$ be the absolute value of the eigenvalues of $F$ for its action on the character group of $\mathbf{T}$. Then $q$ is an integral power of $p$, except in the 'very-twisted cases' giving rise to the Suzuki and Ree groups, where it is an half-integral power. The associated set of fixed points $\mathbf{G}(q):=\mathbf{G}^{F}$ is called a finite group of Lie type.

Now Tits's Theorem, see [31, Theorem 24.17], says that except in the cases

$$
\mathrm{SL}_{2}(2), \mathrm{SL}_{2}(3), \mathrm{SU}_{3}(2), \mathrm{Sp}_{4}(2), G_{2}(2),{ }^{2} B_{2}(\sqrt{2}),{ }^{2} G_{2}(\sqrt{3}),{ }^{2} F_{4}(\sqrt{2})
$$


the group $G:=\mathbf{G}(q)$ is perfect, implying that $G$ is quasi-simple, that is $G / Z(G)$ is non-abelian simple; note that $Z(G)$ always is a $p^{\prime}$-group. In this case, $G$ is called a quasi-simple finite group of Lie type.

The non-solvable groups amongst the above exceptions, that is

$$
\mathrm{Sp}_{4}(2), G_{2}(2),{ }^{2} G_{2}(\sqrt{3}),{ }^{2} F_{4}(\sqrt{2}),
$$

all turn out to have trivial center, and their derived subgroups have index $p$ and are non-abelian simple. These groups are called the almost simple finite groups of Lie type. In both the quasi-simple and the almost simple cases the group $(G / Z(G))^{\prime}$ is called a simple group of Lie type; note that this encompasses the Tits group ${ }^{2} F_{4}(\sqrt{2})^{\prime}$, which does not occur elsewhere in the classification of finite simple groups.

Proposition 2.2. Finite and tame cases; see also [13, Section 8.9]. Let $G$ be a simple or an almost simple finite group of Lie type, and let $U \leq G$ be a Sylow p-subgroup of $G$. Then the following holds:

(a) If $p$ is arbitrary, then $U$ is cyclic if and only if one of the following holds:

○ $G \cong \mathrm{SL}_{2}(p)$, in which case $U \cong C_{p}$ has order $p$;

○ $G \cong{ }^{2} G_{2}(\sqrt{3})^{\prime}$, in which case $U \cong C_{9}$ has order 9 .

(b) If $p=2$, then $U$ is dihedral, semi-dihedral or generalized quaternion if and only if one of the following holds:

- $G \cong \mathrm{SL}_{2}(4)$, in which case we have $U \cong C_{2}^{2}$, the Klein 4-group;

○ $G \cong \mathrm{SL}_{3}(2)$, in which case we have $U \cong D_{8}$, the dihedral group of order 8 ;

○ $G \cong \operatorname{Sp}_{4}(2)^{\prime}$, in which case we have $U \cong D_{8}$, the dihedral group of order 8 .

Proof. Although there is a thorough discussion of the structure of $U$ in [11, Section 3.3 , we choose a direct approach, tailored for our purposes. The almost simple groups and their derived subgroups being discussed in 2.8 below, we may assume that $G=\mathbf{G}^{F}$ is quasi-simple, and that $U:=\mathbf{U}^{F}$, by [31, Corollary 24.11].

(a) Assume that $U$ is cyclic. Then it follows from [31, Proposition 23.7, Corollary 23.9] that $G$ has twisted Lie rank 1, thus $U$ is a root subgroup. For the structure of the root subgroups occurring we refer to [31, Example 23.10] and [5, Proposition 13.6.3, Proposition 13.6.4]. We now consider the various types:

For type $A_{1}$ we have $G \cong \mathrm{SL}_{2}(q)$ and $U \cong \mathbb{F}_{q}^{+}$, hence $U$ is cyclic if and only if $q=p$. For type ${ }^{2} A_{2}$ we have $G \cong \mathrm{SU}_{3}(q)$ and $U / U^{\prime} \cong \mathbb{F}_{q^{2}}^{+}$, hence $U$ is not cyclic. For types ${ }^{2} B_{2}$ and ${ }^{2} G_{2}$ we have $q=\sqrt{2}^{2 f+1}$ and $q=\sqrt{3}^{2 f+1}$, respectively, for some $f \geq 1$, and $U / U^{\prime} \cong \mathbb{F}_{q^{2}}^{+}$, hence $U$ is not cyclic either.

(b) Let $p=2$, and assume that $U$ is dihedral, semi-dihedral or generalized quaternion. Then, by [14, Satz I.14.9(b)], we have $U / U^{\prime} \cong C_{p}^{2}$. Thus the Chevalley commutator formula, see 31, Proposition 23.11], shows that $G$ has has twisted Lie rank at most 2 . We first consider the various types of Lie rank 1 :

For type $A_{1}$ the subgroup $U \cong \mathbb{F}_{q}^{+}$has the desired shape if and only if $q=4$. For type ${ }^{2} A_{2}$ we have $U / U^{\prime} \cong \mathbb{F}_{q^{2}}^{+}$, where $q>2$, hence $U$ does not have the desired shape. For type ${ }^{2} B_{2}$ we have $U / U^{\prime} \cong \mathbb{F}_{q^{2}}^{+}$, where $q^{2}=2^{2 f+1}>2$, hence $U$ does not have the desired shape either.

If $G$ has twisted Lie rank 2 , then $U / U^{\prime}$ has a quotient isomorphic to $\left(U_{\alpha} / U_{\alpha}^{\prime}\right) \times$ $\left(U_{\beta} / U_{\beta}^{\prime}\right)$, where $\alpha, \beta$ denote the fundamental roots, and we assume $\alpha$ to be the long one if there are two distinct root lengths. Hence we have $U_{\alpha} / U_{\alpha}^{\prime} \cong C_{p} \cong U_{\beta} / U_{\beta}^{\prime}$, implying that $U_{\alpha} \cong C_{p} \cong U_{\beta}$. We again consider the various types: 
For type $A_{2}$ we have $G \cong \operatorname{SL}_{3}(q)$ and $U_{\alpha} \cong \mathbb{F}_{q}^{+} \cong U_{\beta}$, hence $q=2$, in which case from $U^{\prime}=Z(U)=U_{\alpha+\beta} \cong \mathbb{F}_{q}^{+}$we get $U \cong D_{8}$. For types $B_{2}$ and $G_{2}$, where $B_{2}(q) \cong \operatorname{Sp}_{4}(q)$, we have $U_{\alpha} \cong \mathbb{F}_{q}^{+} \cong U_{\beta}$, where $q>2$, hence $U$ does not have the desired shape. For type ${ }^{2} A_{3}$ we have $G \cong \mathrm{SU}_{4}(q)$, where $U_{\alpha} \cong \mathbb{F}_{q}^{+}$and $U_{\beta} \cong \mathbb{F}_{q^{2}}^{+}$. For type ${ }^{2} A_{4}$ we have $G \cong \mathrm{SU}_{5}(q)$, where $U_{\alpha} \cong \mathbb{F}_{q^{2}}^{+}$and $U_{\beta} / U_{\beta}^{\prime} \cong \mathbb{F}_{q^{2}}^{+}$. For type ${ }^{3} D_{4}$ we have $U_{\alpha} \cong \mathbb{F}_{q}^{+}$and $U_{\beta} \cong \mathbb{F}_{q^{3}}^{+}$. For type ${ }^{2} F_{4}$ we have $q=\sqrt{2}^{2 f+1}$, for some $f \geq 1$, and $U_{\alpha} \cong \mathbb{F}_{q^{2}}^{+}$and $U_{\beta} / U_{\beta}^{\prime} \cong \mathbb{F}_{q^{2}}^{+}$.

2.3. Blocks. Given a finite group $G$ of Lie type, then the $p$-blocks of $k G$ are wellunderstood; see for example [13, Chapter 8] or [7, Corollary]: There is a unique $p$-block of defect 0 , its ordinary character being the Steinberg character. All other $p$-blocks have maximal defect, and if $k$ contains $|Z(G)|$-th primitive roots of unity, there are precisely $|Z(G)|$ of them. In particular, the principal $p$-block is the only block of positive defect of a simple or an almost simple finite group of Lie type.

Moreover, excluding the cases explicitly mentioned in Proposition 2.2 it follows from the comments in 2.4 below that any block algebra of $k G$ of positive defect has wild representation type.

2.4. Auslander-Reiten theory. We recall the necessary facts from AuslanderReiten theory. Let $G$ be any finite group, and let $B$ be a $p$-block algebra of $k G$, where $k$ is assumed to be algebraically closed. Then it is well-known, see for example [3. Theorem 4.4.4], that $B$ has wild representation type if and only if the defect groups of $B$ are neither cyclic, dihedral, semi-dihedral nor generalized quaternion.

In this case, by [9, Theorem 1], any connected component of the stable AuslanderReiten quiver of $B$ has tree class $A_{\infty}$. Hence it makes sense to discuss whether or not a non-projective indecomposable $B$-module $M$ lies at the end of its connected component in the stable Auslander-Reiten quiver; see for example [18, Section 2.2] or [19, Introduction]. As the Heller operator $\Omega$ induces an automorphism of the stable Auslander-Reiten quiver of $B$, the module $M$ lies at the end of its connected component in the stable Auslander-Reiten quiver, if and only if any and hence all of its Heller translates $\Omega^{i}(M)$, for $i \in \mathbb{Z}$, have this property.

In particular, if $S$ is a simple $B$-module, then the Auslander-Reiten sequence ending in $\Omega^{-1}(S)$ is the standard short exact sequence

$$
\{0\} \rightarrow \Omega(S) \rightarrow P(S) \oplus \mathcal{H}(S) \rightarrow \Omega^{-1}(S) \rightarrow\{0\},
$$

where $\mathcal{H}(S):=\operatorname{rad}(P(S)) / \operatorname{soc}(P(S))$ is the heart of $P(S)$. Hence $S$ lies at the end of its connected component in the stable Auslander-Reiten quiver, if and only if $\mathcal{H}(S)$ is indecomposable.

Another sufficient condition to ensure that $S$ lies at the end of its connected component in the stable Auslander-Reiten quiver is given in the following Proposition 2.5. Actually, it is the key ingredient in the proof of Theorem 2.7 and its immediate Corollary 2.6 is used in 2.8 below:

Proposition 2.5. 20, Proposition 2.1]. Let $G$ be a finite group, and let $U \leq G$ be a Sylow p-subgroup, which is neither cyclic, dihedral, semi-dihedral nor generalized quaternion. Let $S$ be a simple $k G$-module, such that $U$ is a vertex of $S$, and

$$
\operatorname{dim}_{k}\left(\operatorname{Hom}_{k U}\left(k_{U}, S \downarrow_{U}\right)\right)=1=\operatorname{dim}_{k}\left(\operatorname{Hom}_{k U}\left(S \downarrow_{U}, k_{U}\right)\right) .
$$

Then $S$ lies at the end of its connected component in the stable Auslander-Reiten quiver of $k G$. 
Proof. By assumption, $k U$ has wild representation type. Moreover, the second condition on $S$ implies that $S \downarrow_{U}$ is indecomposable. Now the assertion follows from the argument in [20, Section 3] in conjunction with [20, Proposition 1.3].

Corollary 2.6. Keeping the assumptions on $G$ and $U$, let $S$ be a simple $k G$-module.

(a) Let $\Phi(U)$ be the Frattini subgroup of $U$. Suppose that $S_{\downarrow \Phi(U)}$ is indecomposable, and that

$$
\operatorname{dim}_{k}\left(\operatorname{Hom}_{k U}\left(k_{U}, S \downarrow_{U}\right)\right)=1=\operatorname{dim}_{k}\left(\operatorname{Hom}_{k U}\left(S \downarrow_{U}, k_{U}\right)\right) .
$$

Then $S$ lies at the end of its connected component in the stable Auslander-Reiten quiver of $k G$.

(b) Let $H$ be a finite group containing $G$ as a normal subgroup, such that $S$ extends to a (simple) $k H$-module $T$. Moreover, assume that $S$ fulfills the conditions in (a). Then $T$ lies at the end of its connected component in the stable AuslanderReiten quiver of $k H$.

Proof. (a) It follows from the second condition that $S \downarrow_{U}$ is indecomposable. Assume there is a maximal subgroup $X<U$ such that $S \downarrow_{U}$ is relatively $X$-projective. Then $S \downarrow_{U}$ is a direct summand of $\left(S \downarrow_{X}\right) \uparrow^{U}$. From $\Phi(U)<X$ we get that $S \downarrow_{X}$ is indecomposable, hence by Green's indecomposability theorem we infer that $\left(S \downarrow_{X}\right) \uparrow^{U}$ is indecomposable. Thus we have $S \downarrow_{U} \cong\left(S \downarrow_{X}\right) \uparrow^{U}$ as $k U$-modules, a contradiction. Hence $U$ is a vertex of $S$, and the assertion follows from Proposition 2.5.

(b) Let $V$ be a Sylow $p$-subgroup of $H$, where we may assume that $U=G \cap V$. Then we have $U \unlhd V$, and thus by [14, Hilfssatz III.3.3(b)] we get $\Phi(U) \unlhd \Phi(V)$, implying that $T \downarrow_{\Phi(V)}$ is indecomposable. Moreover, since $\operatorname{Hom}_{k V}\left(k_{V}, T \downarrow_{V}\right) \neq\{0\} \neq$ $\operatorname{Hom}_{k V}\left(T \downarrow_{V}, k_{V}\right)$ anyway, from the assumptions on $S$ we infer that

$$
\operatorname{dim}_{k}\left(\operatorname{Hom}_{k V}\left(k_{V}, T \downarrow_{V}\right)\right)=1=\operatorname{dim}_{k}\left(\operatorname{Hom}_{k V}\left(T \downarrow_{V}, k_{V}\right)\right) .
$$

Hence the assertion follows from (a), applied to the $k H$-module $T$.

The key to prove our main theorem will be the following result:

Theorem 2.7. Kawata-Michler-Uno 20, Theorem]. Let $G$ be a quasi-simple, an almost-simple or a simple finite group of Lie type (in the sense of 2.1), defined over a finite field of characteristic $p$, such that its Sylow p-subgroups are neither cyclic, dihedral, semi-dihedral nor generalized quaternion, that is

$$
G \notin\left\{\mathrm{SL}_{2}(p), \mathrm{SL}_{2}(4), \mathrm{SL}_{3}(2), \mathrm{Sp}_{4}(2)^{\prime},{ }^{2} G_{2}(\sqrt{3})^{\prime}\right\} .
$$

Then any non-projective simple $k G$-module, where $k$ is algebraically closed, lies at the end of its connected component in the stable Auslander-Reiten quiver.

Proof. Some care in applying in [20, Section 3] has to be excercised: The strategy of proof is to ensure that the conditions listed in Proposition 2.5 are fulfilled. These in turn follow from the results in [6, 7, including their proofs. Now checking the assumptions made there it turns out that the admissible groups are precisely the quasi-simple groups (in the sense of 2.1). Hence this covers the quasi-simple cases, and it remains to consider the almost simple groups and their derived subgroups, which is done in 2.8 below.

2.8. The lost cases. We discuss the cases excluded in Theorem 1.1 and the Kawata-Michler-Uno Theorem 2.7, and show that indeed the statements do not 
hold in these cases. Moreover, we complete the proof of Theorem 2.7 by considering the relevant almost simple groups and their derived subgroups.

In order to proceed, we assume $k$ to be algebraically closed. Given a finite group $G$, by [37, Theorem E] the heart $\mathcal{H}\left(k_{G}\right)$ of $P\left(k_{G}\right)$ is decomposable if and only if $p=2$ and the Sylow $p$-subgroups of $G$ are dihedral, including the Klein 4-group. In this case, there are three simple $k G$-modules belonging to the principal 2-block, and the structure of the associated projective indecomposable modules has been determined in [8, Theorems 2 and 4]. If the Sylow $p$-subgroups of $G$ are cyclic, then the theory of blocks cyclic defect applies, and the projective indecomposable $k G$-modules are described in terms of the Brauer trees of $k G$; see [17 or 27.

In contrast to this bright picture, for block algebras of wild representation type general theory does not provide too much insight. But the projective indecomposable modules of interest here are straightforwardly constructed explicitly and analysed, using the computer algebra systems GAP [10] and C-MeatAxe [35, and the databases compiled in the framework of the ModularAtlas project [16. The only exceptions are the group ${ }^{2} F_{4}(\sqrt{2})$ and its derived subroup, the Tits group ${ }^{2} F_{4}(\sqrt{2})^{\prime}$, where the projective indecomposable modules are not easily computationally tractable due to sheer size. Here we instead apply Corollary 2.6 to the simple modules in question. Note that restriction induces a bijection between the simple modules in the principal $p$-blocks of an almost simple group and its derived subgroup, respectively. We now consider the various cases:

○ For $G=\mathrm{SL}_{2}(p)$, where $p \geq 5$, the Sylow $p$-subgroups are cyclic of order $p$. There are $\frac{p-1}{2}$ simple modules in each of the two $p$-blocks of positive defect. Their Brauer trees are straight lines having the exceptional vertex of multiplicity 2 at the end, see [13, Section 16.9]. Thus there are two simple modules, amongst them $k_{G}$, such that the heart of the associated projective cover is indecomposable, and $p-3$ simple modules not having this property. Moreover, we have $\operatorname{LL}\left(P\left(k_{G}\right)\right)=3$.

○ For $G={ }^{2} G_{2}(\sqrt{3}) \cong \mathrm{PSL}_{2}(8): 3$ the Sylow 3 -subgroups are isomorphic to the extra-special group $3_{-}^{1+2}$, those of $G^{\prime}={ }^{2} G_{2}(3)^{\prime} \cong \mathrm{SL}_{2}(8) \cong \mathrm{PSL}_{2}(8)$ are isomorphic to $C_{9}$. There are two simple modules $\left\{k_{G}, 7\right\}$ in the principal 3-block of $G^{\prime}$. The assocoated Brauer tree is a straight line having the exceptional vertex of multiplicity 4 at the end. Thus $\mathcal{H}\left(k_{G^{\prime}}\right)$ is indecomposable and $\operatorname{LL}\left(P\left(k_{G^{\prime}}\right)\right)=3$, while $\mathcal{H}(7)$ is decomposable and $\operatorname{LL}(P(7))=5$. The principal 3-block of $G$ has wild representation type, and $\mathcal{H}(S)$ turns out to be indecomposable for both its simple modules $S \in\left\{k_{G}, 7\right\}$, where $\operatorname{LL}\left(P\left(k_{G}\right)\right)=5$ and $\operatorname{LL}(P(7))=7$.

○ For $G=\mathrm{SL}_{2}(4)=\mathrm{PSL}_{2}(4) \cong \mathrm{PSL}_{2}(5)$ and for $G=\mathrm{SL}_{3}(2)=\mathrm{PSL}_{3}(2) \cong$ $\mathrm{PSL}_{2}(7)$ the Sylow 2-subgroups are dihedral, so that $\mathcal{H}\left(k_{G}\right)$ is decomposable.

○ For $G=\operatorname{Sp}_{4}(2) \cong \mathfrak{S}_{6}$ the Sylow 2-subgroups are isomorphic to $D_{8} \times C_{2}$, those of $G^{\prime}=\operatorname{Sp}_{4}(2)^{\prime} \cong \mathfrak{A}_{6} \cong \operatorname{PSL}_{2}(9)$ are isomorphic to $D_{8}$. Hence $P\left(k_{G^{\prime}}\right)$ is decomposable. The principal 2-block of $G$ has wild representation type, and for all its simple modules $S \in\left\{k_{G}, 4,4^{\prime}\right\}$ the heart $\mathcal{H}(S)$ turns out to be indecomposable, where $\operatorname{LL}(P(S))=10$.

- For $G=G_{2}(2) \cong \operatorname{PSU}_{3}(3): 2$ the Sylow 2-subgroups have order 64, those of $G^{\prime} \cong G_{2}(2)^{\prime} \cong \operatorname{PSU}_{3}(3) \cong \mathrm{SU}_{3}(3)$ have order 32 . In both cases they have nilpotency class 3, thus by [14, Satz III.11.9(b)] they are neither cyclic, dihedral, semi-dihedral nor generalized quaternion. Hence both the principal 2-blocks of $G^{\prime}$ and $G$ have wild representation type. For all the simple $k G^{\prime}$-modules $S \in\left\{k_{G^{\prime}}, 6,14\right\}$ the heart $\mathcal{H}(S)$ turns out to be indecomposable, where $\operatorname{LL}(P(S))=19$, and similarly for all the 
simple $k G$-modules $S \in\left\{k_{G}, 6,14\right\}$ the heart $\mathcal{H}(S)$ turns out to be indecomposable, where $\operatorname{LL}(P(S))=20$.

○ For $G \cong{ }^{2} F_{4}(\sqrt{2})$ the Sylow 2 -subgroups have order 4096, those of ${ }^{2} F_{4}(\sqrt{2})^{\prime}$ have order 2048. In both cases they have nilpotency class 8 , thus they are neither cyclic, dihedral, semi-dihedral nor generalized quaternion. Hence both the principal 2-blocks of $G^{\prime}$ and $G$ have wild representation type. Due to sheer size, in these cases the projective indecomposable modules are not easily computationally tractable, so that here we revert to Corollary [2.6] instead. By 2.6(b) it suffices to check the conditions of 2.6(a) for the simple $k G^{\prime}$-modules, which is straightforwardly done explicitly. This shows that for all the simple $k G^{\prime}$-modules or $k G$-modules $S$ the heart $\mathcal{H}(S)$ is indecomposable, but does not provide any further information on the Loewy lengths of the associated projective indecomposable modules. Actually, with considerable computational effort it is possible to show that for the simple $k G^{\prime}$-modules $\left\{k_{G^{\prime}}, 26,246\right\}$ we have $\operatorname{LL}\left(P\left(k_{G^{\prime}}\right)\right)=34$ and $\operatorname{LL}(P(26))=40=\operatorname{LL}(P(246))$, and for the simple $k G$-modules $S \in\left\{k_{G}, 26,246\right\}$ we similarly have $\operatorname{LL}\left(P\left(k_{G^{\prime}}\right)\right)=35$ and $\operatorname{LL}(P(26))=41=\operatorname{LL}(P(246))$.

\section{Proofs}

We are now prepared to prove the results announced in the introduction. To do so, we will need an easy lemma, which we prove explicitly for convenience:

Lemma 3.1. Let $\iota: N \rightarrow M$ be an embedding of A-modules, such that

$$
\operatorname{Hom}_{A}(N / \operatorname{rad}(N), M /(\iota(N)+\operatorname{rad}(M)))=\{0\},
$$

that is the heads of $N$ and $M / \iota(N)$ do not have any composition factors in common. Then any epimorphism $\pi: M \rightarrow N$ is split, and $\iota$ is a splitting map.

Proof. Assume that $\iota(N)+\operatorname{ker}(\pi) \lesseqgtr M$. Then there is a maximal $A$-submodule $L \lesseqgtr M$ containing $\iota(N)+\operatorname{ker}(\pi)$. Hence the simple $A$-module $M / L$ is an epimorphic image both of $M / \iota(N)$ and of $M / \operatorname{ker}(\pi) \cong N$, contradicting the assumption on composition factors. Hence we have $\iota(N)+\operatorname{ker}(\pi)=M$. Now we get $\operatorname{dim}_{k}(\iota(N))+$ $\operatorname{dim}_{k}(\operatorname{ker}(\pi))=\operatorname{dim}_{k}(N)+\left(\operatorname{dim}_{k}(M)-\operatorname{dim}_{k}(N)\right)=\operatorname{dim}_{k}(M)$, implying $\iota(N) \cap$ $\operatorname{ker}(\pi)=\{0\}$, and thus $M=\iota(N) \oplus \operatorname{ker}(\pi)$ as $A$-modules.

Our standard application of Lemma 3.1 will be the following: Let $G$ be a finite group, and let $N \leq M$ be self-dual $k G$-modules, such that even $N$ and $M / N$ do not have any composition factors in common. Then dualising the natural inclusion map $\iota: N \rightarrow M$ gives rise to an epimorphism $\pi: M \cong M^{*} \rightarrow N^{*} \cong N$, and hence we have $M \cong N \oplus(M / N)$ as $k G$-modules

The proofs of Theorems 1.1 and 1.3 will both be based on the following statement:

Proposition 3.2. Let $p$ be odd, let $G$ be a finite group such that $p|| G \mid$, and assume that $\operatorname{LL}\left(P\left(k_{G}\right)\right) \leq 4$. Then we have $\operatorname{LL}\left(P\left(k_{G}\right)\right)=3$, where the heart

$$
\mathcal{H}\left(k_{G}\right):=\operatorname{rad}\left(P\left(k_{G}\right)\right) / \operatorname{soc}\left(P\left(k_{G}\right)\right)
$$

of $P\left(k_{G}\right)$ is simple. Writing $S:=\mathcal{H}\left(k_{G}\right)$ the following holds:

(i) If $S \cong k_{G}$, then we have $p=3$, and $G$ is 3-nilpotent with Sylow 3-subgroups of order 3 .

(ii) If $S \neq k_{G}$, letting $\mathcal{H}(S):=\operatorname{rad}(P(S)) / \operatorname{soc}(P(S))$ be the heart of $P(S)$, then 
$\circ$ either we have $p=3$, and $G$ is not 3-nilpotent, has Sylow 3-subgroups of order 3 , and $\mathcal{H}(S) \cong k_{G}$,

- or $\mathcal{H}(S)$ is strictly decomposable having $k_{G}$ as a direct summand.

Proof. Since $\operatorname{rad}(\tilde{k} G)=\operatorname{rad}(k G) \otimes_{k} \tilde{k}$ for any separable field extension $k \subseteq \tilde{k}$, implying that $\operatorname{LL}\left(P\left(k_{G}\right)\right)=\operatorname{LL}\left(P\left(\tilde{k}_{G}\right)\right)$, we can assume that $k$ is algebraically closed; see also [23, Lemma 1.6 and its proof].

By Maschke's Theorem, we have $\operatorname{LL}\left(P\left(k_{G}\right)\right) \geq 2$. Suppose that $\operatorname{LL}\left(P\left(k_{G}\right)\right)=2$, then $k_{G}$ is the only simple $k G$-module in $B_{0}(G)$, and from the Cartan invariant $c_{11}(G)=\left[P\left(k_{G}\right): k_{G}\right]=2$ we conclude that $p=2$, a contradiction. Similarly, it follows from [21, Corollary] that $\operatorname{LL}\left(P\left(k_{G}\right)\right) \neq 4$.

Hence we have $\operatorname{LL}\left(P\left(k_{G}\right)\right)=3$. Thus $\mathcal{H}\left(k_{G}\right)$ is semi-simple, hence is simple by 37. Theorem E], so we write $S:=\mathcal{H}\left(k_{G}\right)$. If $S \cong k_{G}$, then $k_{G}$ is the only simple $k G$-module in $B_{0}(G)$, hence by Brauer's Theorem [33, Theorem V.8.3] we conclude that $G$ is $p$-nilpotent, and from $c_{11}(G)=\left[P\left(k_{G}\right): k_{G}\right]=3$ we conclude that $p=3$ and that the Sylow $p$-subgroups of $G$ have order 3 .

Thus we may assume that $S \not k_{G}$, and let $\mathcal{H}(S):=\operatorname{rad}(P(S)) / \operatorname{soc}(P(S))$. Since $1=c_{1, S}(G)=\left[P\left(k_{G}\right): S\right]=\left[P(S): k_{G}\right]=c_{S, 1}(G)$, and since there is a uniserial $k G$ module with composition factors $S$ and $k_{G}$, Lemma 3.1]implies that $\mathcal{H}(S)=k_{G} \oplus \mathcal{H}^{\prime}$ for a $k G$-module $\mathcal{H}^{\prime}$.

Assume that $\mathcal{H}^{\prime}=\{0\}$. Then $\left\{k_{G}, S\right\}$ are the simple $k G$-modules in $B_{0}(G)$, and thus the Cartan matrix $C$ of $B_{0}(G)$ is of the form

$$
C=\left(\begin{array}{ll}
2 & 1 \\
1 & 2
\end{array}\right) .
$$

Hence we have $\operatorname{det}(C)=3$, thus the Sylow $p$-subgroups of $G$ have order 3. Moreover, it follows from the theory of blocks of cyclic defect that the inertial index of $B_{0}(G)$ is 2 , and thus $G$ is not 3 -nilpotent. Therefore we are left with the case $\mathcal{H}^{\prime} \neq\{0\}$, that is $\mathcal{H}(S)$ is decomposable of the desired form.

Proof of Theorem 1.1. Assume to the contrary that $\operatorname{LL}\left(P\left(k_{G}\right)\right) \leq 4$. Since by Proposition 2.2 we are assuming that $G$ has non-cyclic Sylow $p$-subgroups, from Proposition 3.2 we infer the existence of a simple $k G$-module $S \not k_{G}$, such that $\mathcal{H}(S)$ is decomposable. By 2.4 this means that $S$ does not lie at the end of its connected component in the stable Auslander-Reiten quiver, contradicting the KawataMichler-Uno Theorem 2.7.

Proof of Corollary 1.2, This follows immediately from Theorem 1.1.

Proof of Theorem 1.3. Set $H:=O^{p^{\prime}}(G)$; note that $p|| H \mid$. Then we have $O^{p^{\prime}}(H)=H$, and $H \leq G$ being a characteristic subgroup, from $O_{p^{\prime}}(G)=\{1\}$ we infer $O_{p^{\prime}}(H)=\{1\}$.

By [26, Lemma 4.1] we have $\operatorname{LL}\left(B_{0}(H)\right)=4$, where $B_{0}(H)$ is the principal block algebra of $k H$. Hence we have $\operatorname{LL}\left(P\left(k_{H}\right)\right) \leq 4$, and thus from Proposition 3.2 we get that the heart $S:=\mathcal{H}\left(k_{H}\right)$ of $P\left(k_{H}\right)$ is simple; see also [24, Proposition 4.6]. Moreover, if $S \cong k_{H}$, then from $O_{p^{\prime}}(H)=\{1\}$ we conclude that $H \cong C_{3}$, hence $\mathrm{LL}\left(B_{0}(H)\right)=\operatorname{LL}\left(P\left(k_{H}\right)\right)=3$, a contradiction. Hence we have $S ¥ k_{H}$, so that the Cartan invariant $c_{11}(H)=2$.

We are prepared to show that $H$ is non-abelian simple: Suppose that there exists $N$ such that $\{1\} \neq N \triangleleft H$. Then from $O_{p^{\prime}}(H)=\{1\}$ and $O^{p^{\prime}}(H)=H$ we conclude 
that both $p|| N \mid$ and $p|| H / N \mid$, contradicting [22, Lemma 2.5]. Moreover, if $H$ were abelian, then $H \cong C_{p}$, so that $\operatorname{LL}\left(B_{0}(H)\right)=\mathrm{LL}(k H)=p$, a contradiction.

Next we consider the Fitting subgroup $F(G)$. Since by assumption we have $O_{p^{\prime}}(G)=\{1\}$, it remains to consider $O_{p}(G)$. Since $H$ is non-abelian simple we have $O_{p}(G) \cap H=\{1\}$. From this we get $O_{p}(G) \cong O_{p}(G) H / H \leq G / H$, where the latter is a $p^{\prime}$-group, so that $O_{p}(G)=\{1\}$, hence $F(G)=\{1\}$, that is $F^{*}(G)=E(G)$.

Finally we consider the layer $E(G)$. Clearly, as $H$ is a component of $G$, we have $H \leq E(G)$. Hence in order to claim that $H=E(G)$ we have to show that there is no other component of $G$. Suppose to the contrary that there is a component $Q \neq H$ of $G$; note that $Q \not \leq H$. Then let $N:=\left\langle Q^{g} \mid g \in G\right\rangle \unlhd G$ be the normal closure of $Q$ in $G$. Hence we have $N \cap H=\{1\}$, since otherwise, $H$ being simple, we had $N \cap H=H$, that is $Q \leq N \leq H$, a contradiction. From this we get $N \cong N H / H \leq G / H$, so that $N$ is normal $p^{\prime}$-subgroup of $G$, hence $O_{p^{\prime}}(G)=\{1\}$ implies $Q \leq N=\{1\}$, a contradiction.

We end this paper with a concluding remark.

Remark 3.3. 'Minimal' projective indecomposable modules. (a) Given a finite group $G$ and a simple $k G$-module $S$, let $c(S)$ be the integer defined by

$$
c(S):=\frac{\operatorname{dim}_{k}(P(S))}{|G|_{p}},
$$

where $|G|_{p}$ is the largest power of $p$ dividing $|G|$. Following [32, if $c(S)=1$ then the projective indecomposable module $P(S)$ is called minimal.

Now, given a normal subgroup $N \unlhd G$, Malle-Weigel [32, Proposition 2.2] show 'supermultiplicativity' $c\left(k_{G}\right) \geq c\left(k_{N}\right) \cdot c\left(k_{G / N}\right)$, they give sufficient conditions as to when multiplicativity holds, and they ask whether this is possibly always fulfilled.

However, even more generally, given any simple $k G$-module $S$ on which $N$ acts trivially, and denoting its deflation to $G / N$ by $\bar{S}$, it is an immediate consequence of the Alperin-Collins-Sibley Theorem, see [1, Corollary 1], that $c(S)=c\left(k_{N}\right) \cdot c(\bar{S})$; see also [38, Lemma 2.6, Section 4] and [15, Theorem VII.14.2]. In particular, the above question has an affirmative answer.

(b) We are mentioning this, as we are going to indicate two alternative ways to proceed in the final step in the proof of Theorem 1.1 they both need ordinary representation theory. Hence let $G$ be a simple or an almost simple finite group of Lie type, and recall that we know that $S:=\mathcal{H}\left(k_{G}\right)$ is simple, where $S \neq k_{G}$.

(i) Firstly, for the untwisted and twisted cases we have $|U|=q^{N}$, while for the very-twisted cases we have $|U|=\left(q^{2}\right)^{N}$, where $N$ is the number of positive roots in the root system associated with $\mathbf{G}$. We have $\operatorname{dim}_{k}(S)=c\left(k_{G}\right) \cdot|U|-2$, while by [13, Theorem 3.7] and a similar result for the very-twisted cases, we have $\operatorname{dim}_{k}(S)<|U|$. This implies $c\left(k_{G}\right)=1$, that is $P\left(k_{G}\right)$ is minimal, contradicting [32, Theorem 5.8]; to prove the latter ordinary character theory of finite groups of Lie type is used.

(ii) Secondly, more straightforwardly, we observe that $\operatorname{dim}_{k}(S)=|U|-2$. Then, for the untwisted and twisted cases we may infer $N=1$, proceeding similar to [13, Proof of Theorem 3.7], using Weyl's character formula for ordinary Verma modules; see also [28, Proposition 5.1]. Thus we get $G=\mathrm{SL}_{2}(q)$, and hence $\operatorname{dim}_{k}(S)=q-2$. Whence Steinberg's Tensor Product Theorem, see [13, Theorem 2.7], implies $q=p$, a contradiction. For the the very-twisted case ${ }^{2} G_{2}\left(\sqrt{3}^{2 f+1}\right)$ a similar dimension estimate yields a contradiction, see [13, Chapter 20]. 


\section{ACKNOWLEDGMENTS}

A part of this work was done while the first author was visiting University of Jena several times in 2015-2016, while the second author was visiting University of Chiba in 2015, and also while the authors were both visiting the Centre Interfacultaire Bernoulli (CIB) at École Polytechnique Fédérale de Lausanne (EPFL) during the Semester 'Local representation theory and simple groups' in 2016. The authors are grateful to these institutions for their hospitality. The first author was partially supported by the Japan Society for Promotion of Science (JSPS), Grant-in-Aid for Scientific Research (C)15K04776, 20152018; and also by the CIB in EPFL. The second author was financially supported by the German Science Foundation (DFG) Scientific Priority Programme SPP-1478 'Algorithmic and Experimental Methods in Algebra, Geometry, and Number Theory'.

\section{REFERENCES}

[1] J.L. Alperin, M.J. Collins, D.A. Sibley. Projective modules, filtrations and Cartan invariants. Bull. London Math. Soc. 16 (1984), 416-420.

[2] M. Auslander, I. Reiten, S. Smalø. Representation theory of Artin algebras. Corrected reprint of the 1995 original, Cambridge Studies in Advanced Mathematics 36, Cambridge Univ. Press, Cambridge, 1997.

[3] D.J. Benson. Representations and Cohomology I: Basic representation theory of finite groups and associative algebras. Cambridge Univ. Press, Cambridge, 1998.

[4] R. Brauer. Representations of finite groups. In: "Lectures on Modern Mathematics" 1, Edited by T.L. Saaty, 133-175, Wiley, New York, 1963.

[5] R. Carter. Simple groups of Lie type. Reprint of the 1972 original, Wiley Classics Library, Wiley, New York, 1989.

[6] R. Dipper. Vertices of irreducible representations of finite Chevalley groups in the describing characteristic. Math. Z. 175 (1980), 143-159.

[7] R. Dipper. On irreducible modules of twisted groups of Lie type. J. Algebra 81 (1983), 370389.

[8] K. Erdmann. Principal blocks of groups with dihedral Sylow 2-subgroups. Comm. Algebra 5 (1977), 665-694.

[9] K. Erdmann. On Auslander-Reiten components for group algebras. J. Pure Appl. Algebra 104 (1995), 149-160.

[10] The GAP Group. GAP - Groups, Algorithms, and Programming, Version 4.8.4, http:// www.gap-system.org, 2016.

[11] D. Gorenstein, R. Lyons, R. Solomon. The classification of the finite simple groups, Number 3, Almost simple $K$-groups. Mathematical Surveys and Monographs 40.3., American Math. Soc., Providence, RI, 1998.

[12] J.E. Humphreys. Cartan invariants. Bull. London Math. Soc. 17 (1985), 1-14.

[13] J.E. Humphreys. Modular Representations of Finite Groups of Lie Type. London Math. Soc. Lecture Note Series 326, Cambridge, 2006.

[14] B. Huppert. Endliche Gruppen I. Grundlehren der Mathematischen Wissenschaften 134, Springer-Verlag, Berlin-New York, 1967.

[15] B. Huppert, N. Blackburn. Finite groups II. Grundlehren der Mathematischen Wissenschaften 242, Springer-Verlag, Berlin-New York, 1982.

[16] C. Jansen, K. Lux, R. Parker, R. Wilson. An Atlas of Brauer Characters. Clarendon Press, Oxford, 1995.

[17] G.J. Janusz. Indecomposable modules for finite groups. Ann. of Math. 89 (1969), 209-241.

[18] T. Jost. On Specht modules in the Auslander-Reiten quiver. J. Algebra 173 (1995), 281-301.

[19] S. Kawata. On Auslander-Reiten components and simple modules for finite group algebras. Osaka J. Math. 34 (1997), 681-688.

[20] S. Kawata, G.O. Michler, K. Uno. On Auslander-Reiten components and simple modules for finite groups of Lie type. Osaka J. Math. 38 (2001), 21-26.

[21] S. Koshitani. Cartan invariants of group algebras of finite groups. Proc. Amer. Math. Soc. 124 (1996), 2319-2323. 
[22] S. Koshitani. The projective cover of the trivial module over a group algebra of a finite group. Comm. Algebra 42 (2014), 4308-4321.

[23] S. Koshitani. Finite groups whose first Cartan invariants over a field of characteristic two are two. J. Algebra 426 (2015), 259-272.

[24] S. Koshitani, B. Külshammer, B. Sambale. On Loewy lengths of blocks. Math. Proc. Camb. Phil. Soc. 156 (2014), 555-570.

[25] S. Koshitani, N. Kunugi. The principal 3-blocks of the 3-dimensional projective special unitary groups in non-defining characteristic. J. reine angew. Math. 539 (2001), 1-27.

[26] S. Koshitani, H. Miyachi. Donovan conjecture and Loewy length for principal 3-blocks of finite groups with elementary abelian Sylow 3-subgroup of order 9. Comm. Algebra. 29 (2001), $4509-4522$.

[27] H. Kupisch. Unzerlegbare Moduln endlicher Gruppen mit zyklischer $p$-Sylow-Gruppe. Math. Z. 108 (1969), 77-104.

[28] C. Lassueur, G. Malle, E. Schulte. Simple endotrivial modules for quasi-simple groups. J. Reine Angew. Math. 712 (2016), 141-174.

[29] F. Lübeck, G. Malle. A Murnaghan-Nakayama rule for values of unipotent characters in classical groups. Rep. Theory 20 (2016), 139-161.

[30] F. Lübeck, G. Malle. Corrigendum: A Murnaghan-Nakayama rule for values of unipotent characters in classical groups. Preprint, arXiv:1508.01627 4 [math.RT] (October 25th, 2016), https://arxiv.org/abs/1508.01627v4.

[31] G. Malle, D. Testerman. Linear algebraic groups and finite groups of Lie type. Cambridge Studies in Advanced Mathematics 133, Cambridge Univ. Press, Cambridge, 2011.

[32] G. Malle, T. Weigel. Finite groups with minimal 1-PIM. Manuscripta Math. 126 (2008), $315-332$.

[33] H. Nagao, Y. Tsushima. Representations of Finite Groups. Academic Press, New York, 1989.

[34] T. Okuyama. On blocks of finite groups with radical cube zero. Osaka J. Math. 23 (1986), 461-465.

[35] M. Ringe. The C-MeatAxe - Computing with Modular Representations, Version 2.4.24, http://www.math.rwth-aachen.de/ MTX, 2011.

[36] M. Suzuki. Group Theory II. Springer-Verlag, Berlin, 1980.

[37] P.J. Webb. The Auslander-Reiten quiver of a finite group. Math. Z. 179 (1982), 97-121.

[38] W. Willems. On the projectives of a group algebra. Math. Z. 171 (1980), 163-174.

[39] R. Wilson, J. Thackray, R. Parker, F. Noeske, J. Müller, F. Lübeck, C. Jansen, G. Hiss, T. Breuer. The Modular Atlas Project, http://www.math.rwth-aachen.de/ MOC

Department of Mathematics, Graduate School of Science, Chiba University, 1-33 YAYOI-Cho, InAGe-Ku, Chiba 263-8522, JAPAN.

E-mail address: koshitan@math.s.chiba-u.ac.jp

Arbeitsgruppe Algebra und Zahlentheorie, Bergische Universität Wuppertal, GaussStrasse 20, D-42119 Wuppertal, Germany.

E-mail address: juergen.mueller@math.uni-wuppertal.de 